PROCEEDINGS OF THE

AMERICAN MATHEMATICAL SOCIETY

Volume 133, Number 11, Pages 3395-3402

S 0002-9939(05)07809-3

Article electronically published on June 20, 2005

\title{
RIGIDITY OF SOME WEYL MANIFOLDS WITH NONPOSITIVE SECTIONAL CURVATURE
}

\author{
MACIEJ P. WOJTKOWSKI
}

(Communicated by Jon G. Wolfson)

\begin{abstract}
We provide a list of all locally metric Weyl connections with nonpositive sectional curvatures on two types of manifolds, $n$-dimensional tori $\mathbb{T}^{n}$ and $\mathbb{M}^{n}=\mathbb{S}^{1} \times \mathbb{S}^{n-1}$ with the standard conformal structures. For $\mathbb{M}^{n}$ we prove that it carries no other Weyl connections with nonpositive sectional curvatures, locally metric or not. In the case of $\mathbb{T}^{n}$ we prove the same in the more narrow class of integrable connections.
\end{abstract}

\section{WeYl CONNECTIONS With NONPOSITIVE SECTIONAL CURVATURES}

Geodesic flows of Weyl connections (W-flows) are models of thermostatted systems under the action of an external field, GR], [R], W1]. Negative sectional curvature can be naturally defined for Weyl connections, and it leads to some hyperbolic properties of the W-flow, W1]. In the search for homogeneous Weyl manifolds with nonpositive sectional curvature one encounters rigidity phenomena similar to the riemannian case, [AW], [W2] .

On a riemannian manifold $\left(M^{n}, g\right)$ one gets a Weyl connection for every 1-form $\varphi$. A defining property of the Weyl connection is that it is a symmetric linear connection $\widehat{\nabla}$ such that $\widehat{\nabla}_{X} g=-2 \varphi(X) g,[\mathrm{~F}]$. For a given 1-form $\varphi$ the Weyl connection is equal to

$$
\widehat{\nabla}_{X} Y=\nabla_{X} Y+\varphi(Y) X+\varphi(X) Y-\langle X, Y\rangle E,
$$

for any tangent vector fields $X, Y$ on $M$, where $\nabla$ is the Levi-Civita (riemannian) connection of $g, E$ is the tangent vector field dual to $\varphi$ and $\langle\cdot, \cdot\rangle$ denotes the metric $g$. A pair $(g, \varphi)$ defines the same Weyl connection as the pair $\left(e^{-2 U} g, \varphi+d U\right)$, which shows that Weyl connections for closed 1-forms are locally metric (riemannian) connections.

The curvature operator of a Weyl connection

$$
\widehat{R}(X, Y)=\widehat{\nabla}_{X} \widehat{\nabla}_{Y}-\widehat{\nabla}_{Y} \widehat{\nabla}_{X}-\widehat{\nabla}_{[X, Y]}
$$

has the symmetric part, called the distance curvature, $\widehat{R}_{s}(X, Y)=d \varphi(X, Y) I$, where $I$ is the identity operator, $[\mathrm{F}]$. The antisymmetric part of the curvature

Received by the editors April 11, 2003 and, in revised form, April 12, 2004.

2000 Mathematics Subject Classification. Primary 53C99, 37Dxx.

Key words and phrases. Weyl connection, nonpositive sectional curvature.

The author is grateful to Leonid Friedlander, Feliks Przytycki, Don Wang and the referee for their comments. 
operator $\widehat{R}_{a}=\widehat{R}-\widehat{R}_{s}$, called the direction curvature, can be used to define the sectional curvature $\widehat{K}(\Pi)$ of the Weyl connection in the direction of a plane $\Pi$

$$
\widehat{K}(\Pi)=\left\langle\widehat{R}_{a}(X, Y) Y, X\right\rangle,
$$

for any orthonormal basis $\{X, Y\}$ of $\Pi$. These sectional curvatures depend on the choice of the riemannian metric $g$ in the conformal class and not on the Weyl connection alone. However, the sign of sectional curvatures is well defined. In particular if $d \varphi=0$ so that $\hat{\nabla}$ is the locally riemannian connection of the metric $e^{-2 U} g$ for the local function $U$ such that $\varphi+d U=0$, we have that the sectional curvatures $K_{U}(\Pi)$ of this new metric and the Weyl sectional curvatures $\widehat{K}(\Pi)$ are related by

$$
K_{U}(\Pi)=e^{2 U} \widehat{K}(\Pi) .
$$

The Weyl sectional curvatures have a surprisingly transparent expression, [W1]:

$$
\widehat{K}(\Pi)=K(\Pi)-E_{\perp}^{2}-\operatorname{div}_{\Pi} E,
$$

where $K(\Pi)$ is the riemannian sectional curvature in the direction of $\Pi$, the vector field $E_{\perp}$ is the component of $E$ orthogonal to $\Pi$ and $\operatorname{div}_{\Pi} E=\left\langle\nabla_{X} E, X\right\rangle+$ $\left\langle\nabla_{Y} E, Y\right\rangle$, for an orthonormal basis $\{X, Y\}$ of $\Pi$, is the "partial" divergence of the vector field $E$.

In the 2-dimensional case there is only one sectional curvature at a point and the formula (1.3) simplifies to $\widehat{K}(\Pi)=K(\Pi)-\operatorname{div} E$. By the theorem of Gauduchon, G], on a compact manifold there is a unique metric in the conformal class (the Gauduchon gauge) such that $\operatorname{div} E=0$. We conclude that for a compact surface the sectional curvature of the Weyl connection with respect to the Gauduchon gauge is equal to the Gaussian curvature. In particular on the 2-dimensional torus $\mathbb{T}^{2}$, if the sectional curvature of a Weyl connection is nonpositive, then it vanishes everywhere, and all such Weyl connections can be obtained from divergence-free vector fields $E$. In the rest of the paper we will consider only manifolds of dimension $n \geq 3$.

For the higher-dimensional torus $\mathbb{T}^{n}, n \geq 3$, with the standard flat metric, the Weyl connection defined by any constant vector field $E$ has by (1.3) negative sectional curvature at any plane $\Pi$ which does not contain $E$, and zero sectional curvature at any plane $\Pi$ containing $E$. We do not know of any other Weyl connections on the standard torus with nonpositive sectional curvatures.

We will call a Weyl connection on a flat torus (or $\mathbb{R}^{n}$ ) integrable if on the set where the 1-form $\varphi$ does not vanish, the distribution of zero subspaces $\{\varphi=0\}$ is integrable. In particular every locally metric Weyl connection is integrable. The notion of integrability depends on the choice of the metric in the conformal class. We will consider it only with respect to the flat metric.

Theorem 1.1. On the torus $\mathbb{T}^{n}, n \geq 3$, with the flat metric, if an integrable Weyl connection has nonpositive sectional curvatures everywhere, then the defining 1form $\varphi$ is constant (equivalently the dual vector field $E$ is constant).

We conjecture that Theorem 1.1 can be strengthened by allowing all Weyl connections, integrable or not. In Proposition 2.1 we will prove that there are no Weyl connections on $\mathbb{T}^{n}$ with negative sectional curvatures everywhere.

For a fixed positive number $a$ let $D$ be the group of dilations of $\mathbb{R}^{n}$ by $a^{k}, k \in$ $\mathbb{Z}$. Let $\mathbb{M}^{n}=\mathbb{S}^{1} \times \mathbb{S}^{n-1}=\left(\mathbb{R}^{n} \backslash\{0\}\right) / D$ with the standard conformal structure inherited from $\mathbb{R}^{n}$. We define a locally riemannian Weyl connection on $\mathbb{M}^{n}$ by the 
metric on the covering space $\mathbb{R}^{n} \backslash\{0\}$ defined in the spherical coordinates $(r, \theta)$ as $d^{2} s=r^{2 \alpha}\left(d^{2} r+r^{2} d^{2} \theta\right)$. Although for $\alpha \neq-1$ the metric does not project on the quotient manifold $\mathbb{M}^{n}$, its Levi-Civita connection does. The connections for $\alpha$ and $-\alpha-2$ are taken into each other by the inversion. In Section 3 we will calculate that these Weyl connections have nonpositive sectional curvatures for $|\alpha+1| \geq 1$.

Theorem 1.2. The locally metric Weyl connections described above are the only Weyl connections on $\mathbb{M}^{n}, n \geq 3$, with nonpositive sectional curvatures.

It will be shown in Section 3 that in the case $-2<\alpha<0$ there are pairs of points in $\mathbb{R}^{n} \backslash\{0\}$ which are connected by two Weyl geodesics, and in the extreme case of two points lying on one line passing through 0 , on the opposite sides of 0 , there are a continuum of connecting geodesics, two in every plane containing the pair of points. In the case of nonpositive sectional curvatures $(|\alpha+1| \geq 1)$, every two points in $\mathbb{R}^{n} \backslash\{0\}$ are connected by exactly one Weyl geodesic or do not have any connection whatsoever.

\section{NeCESSARY AND SUFFICIENT CONDITIONS FOR NONPOSITIVITY OF SECTIONAL CURVATURES ON $\mathbb{T}^{n}$ AND $\mathbb{M}^{n}$}

We consider the euclidean space $\mathbb{R}^{n}$ with the conformal class defined by the metric and two classes of quotient manifolds, the $n$-dimensional tori $\mathbb{T}^{n}=\mathbb{R}^{n} / \mathbb{Z}^{n}$ and $\mathbb{M}^{n}=\mathbb{S}^{1} \times \mathbb{S}^{n-1}=\left(\mathbb{R}^{n} \backslash\{0\}\right) / D$. In both cases the quotient inherits the conformal class from $\mathbb{R}^{n}$ and we can consider Weyl connections with respect to it. A Weyl connection can be lifted to the covering space and expressed in terms of the standard metric as in (1.1) by choosing a vector field $E$. To define a Weyl connection on the quotient space, the vector field $E$ on $\mathbb{R}^{n}\left(\mathbb{R}^{n} \backslash\{0\}\right)$ must be $\mathbb{Z}^{n}$ periodic in the toral case and it has to satisfy the following equivariance property in the case of $\mathbb{M}^{n}$ :

$$
E(a u)=\frac{1}{a} E(u)
$$

for the coefficient of dilation $a>0$, and any $u \in \mathbb{R}^{n} \backslash\{0\}$. For example, the Weyl connections on $\mathbb{M}^{n}$ introduced in Section 1 are defined by $E(u)=\frac{\alpha}{u^{2}} u=\frac{\alpha}{r} e_{r}$, where $r$ is the radial coordinate and $e_{r}$ is the unit radial vector field.

Proposition 2.1. If the Weyl connection on $\mathbb{T}^{n}$ or $\mathbb{M}^{n}$, defined by a vector field $E$ on $\mathbb{R}^{n}\left(\mathbb{R}^{n} \backslash\{0\}\right)$, has nonpositive sectional curvatures everywhere, then at points where $E$ does not vanish the sectional curvatures in the direction of any plane $\Pi$ containing $E$ are equal to zero, i.e., we have

$$
\widehat{K}(\Pi)=-\left\langle\nabla_{X} E, X\right\rangle-\left\langle\nabla_{Y} E, Y\right\rangle=0,
$$

for any orthonormal frame $X, Y$ in the plane $\Pi$.

Proof. For a plane $\Pi$ containing $E$ we have by (1.3)

$$
\widehat{K}(\Pi)=-\left\langle\nabla_{X} E, X\right\rangle-\left\langle\nabla_{Y} E, Y\right\rangle \leq 0,
$$

for $X=|E|^{-1} E$ and any unit vector $Y$ orthogonal to $E$. Averaging over all such vectors $Y$ we get from $(2.3)$

$$
\begin{aligned}
& -\left\langle\nabla_{X} E, X\right\rangle-\frac{1}{n-1} \operatorname{div} E+\frac{1}{n-1}\left\langle\nabla_{X} E, X\right\rangle \\
& =-\frac{1}{n-1}\left(\operatorname{div} E+(n-2)\left\langle\nabla_{X} E, X\right\rangle\right) \leq 0 .
\end{aligned}
$$


It follows that for the $C^{1}$ vector field $V=|E|^{n-2} E$ we have

$$
\operatorname{div} V=|E|^{n-2} \operatorname{div} E+(n-2)\left\langle\nabla_{X} E, X\right\rangle|E|^{n-2} \geq 0 .
$$

If $\mathcal{F} \subset \mathbb{R}^{n}$ is a fundamental domain for our manifold, then by Stokes' theorem we get that $\int_{\mathcal{F}} \operatorname{div} V=0$. Indeed in the case of $\mathbb{T}^{n}$ it is obvious, and in the case of $\mathbb{M}^{n}$ we apply the Stokes' theorem to the fundamental domain between two concentric spheres and use the fact that by $(2.1), V(a u)=a^{-(n-1)} V(a)$, so that the flux across the boundary vanishes. It follows that $\operatorname{div} V=0$ and the expression in (2.4) vanishes. Since it is the average of the nonnegative function (2.3), then the function (2.3) must vanish as well.

Assuming now that a vector field $E$ does not vanish and satisfies the condition from Proposition 2.1, we will obtain necessary and sufficient conditions for the nonpositivity of sectional curvatures at a point.

Proposition 2.2. At a point where the vector field $E$ does not vanish and the Weyl connection defined by it has zero sectional curvature at any plane containing $E$, all the sectional curvatures are nonpositive if and only if

$$
\left\langle\nabla_{E} E, Y\right\rangle=-\left\langle\nabla_{Y} E, E\right\rangle \quad \text { and } \quad 2\left\langle\nabla_{E} E, E\right\rangle \leq|E|^{4},
$$

for any vector $Y$ orthogonal to $E$.

Proof. Let us consider three mutually orthogonal unit vectors $X=\frac{E}{|E|}, Y_{1}, Y_{2}$. We will calculate the sectional curvature in the direction of the plane $\Pi$ spanned by $Y_{1}$ and $a X+b Y_{2}, a^{2}+b^{2}=1$. We have by (1.3) and in view of (2.2)

$$
\begin{aligned}
\widehat{K}(\Pi)= & -E^{2}+a^{2} E^{2}-\left\langle\nabla_{Y_{1}} E, Y_{1}\right\rangle-a^{2}\left\langle\nabla_{X} E, X\right\rangle-b^{2}\left\langle\nabla_{Y_{2}} E, Y_{2}\right\rangle \\
& -a b\left(\left\langle\nabla_{X} E, Y_{2}\right\rangle+\left\langle\nabla_{Y_{2}} E, X\right\rangle\right) \\
=-b^{2} & \left(E^{2}-2\left\langle\nabla_{X} E, X\right\rangle\right)-a b\left(\left\langle\nabla_{X} E, Y_{2}\right\rangle+\left\langle\nabla_{Y_{2}} E, X\right\rangle\right) \leq 0 .
\end{aligned}
$$

\section{The Class OF Weyl CONNECTIONS ON $\mathbb{M}^{n}$ DEFINED BY RADIAL VECTOR FIELDS}

Let us consider an arbitrary radial vector field $E(u)=\frac{\alpha(u)}{u^{2}} u$ where $\alpha$ is a smooth function satisfying $\alpha(a u)=\alpha(u)$, so that (2.1) holds. We want to find all such vector fields which define Weyl connections on $\mathbb{M}^{n}$ with nonpositive sectional curvatures.

The Weyl connection defined by $E$ satisfies the condition from Proposition 2.1 (i.e., in the open set where $E$ does not vanish, it has zero sectional curvature at any plane containing $E$ ) if and only if $\alpha$ is homogeneous of degree 0 (i.e., $\alpha(k u)=\alpha(u)$ for any $k>0$ ). Indeed, we have for any vector field $Z$

$$
\begin{aligned}
& \nabla_{Z} E=\frac{\alpha}{u^{2}} Z+\frac{d \alpha(Z)}{u^{2}} u-\frac{2 \alpha\langle Z, u\rangle}{u^{4}} u, \\
& \left\langle\nabla_{X} E, X\right\rangle=\frac{d \alpha(X)}{|u|}-\frac{\alpha}{u^{2}}, \text { for } X=\frac{u}{|u|}, \\
& \left\langle\nabla_{Y} E, Y\right\rangle=\frac{\alpha}{u^{2}}, \text { for any unit vector } Y \text { orthogonal to } E .
\end{aligned}
$$

We conclude that $(2.2)$ is equivalent to $d \alpha(E)=0$, i.e., where $\alpha$ does not vanish, it is constant on rays. Further, if $\alpha$ vanishes at a point, then it must also vanish on the whole ray passing through it. 
We will now apply Proposition 2.2 to find all radial vector fields $E$ that give rise to Weyl connections with nonpositive sectional curvatures. We have for $X, Y$ as above

$$
\left\langle\nabla_{X} E, Y\right\rangle=0, \quad\left\langle\nabla_{Y} E, X\right\rangle=\frac{d \alpha(Y)}{|u|},
$$

which leads us to the conclusion that $d \alpha(Y)=0$ for any $Y$ orthogonal to $E$. Hence $\alpha=$ const. Finally

$$
E^{2}-2\left\langle\nabla_{X} E, X\right\rangle=\frac{\alpha^{2}}{u^{2}}+\frac{2 \alpha}{u^{2}},
$$

and we conclude that all sectional curvatures of the Weyl connection defined by $E$ are nonpositive if and only if $\alpha$ is constant, and $\alpha \leq-2$ or $\alpha \geq 0$.

These Weyl connections for different constant $\alpha$ seem to be quite similar. The geometric difference between the cases of nonpositive and nonnegative sectional curvatures transpires in the behavior of Weyl geodesics.

The equations of Weyl geodesics in $\mathbb{R}^{n} \backslash\{0\}$ parametrized by the arc length coincide with the equations of the Gaussian thermostat, [W1],

$$
\frac{d u}{d t}=v, \quad \frac{d v}{d t}=E-\langle E, v\rangle v, \quad v^{2}=1 .
$$

It is easy to see that every trajectory of (3.2) lies in a plane passing through 0 and hence the integration of (3.2) reduces to the case $n=2$, which coincides with the toral case. It was observed in [W1] (Proposition 6.5) that for the vector field $E(u)=\frac{\alpha}{u^{2}} u, \alpha \neq-1$, the conformal mapping $z \rightarrow z^{l}, l=\alpha+1$, takes the trajectories of (3.2) into straight lines. Hence in polar coordinates $(r, \theta)$ in $\mathbb{R}^{2} \backslash\{0\}$ the Weyl geodesics are given by the equation $r^{l} \cos l\left(\theta-\theta_{0}\right)=$ const $\neq 0$, or are rays $\theta=$ const. In the case of $\alpha=-1$ it is the conformal mapping $z \rightarrow \ln z$ which takes the trajectories into straight lines and hence the Weyl geodesics are logarithmic spirals or circles centered at the origin.

The inversion of the plane changes $l$ into $-l$. Hence it is sufficient to study the case of $l \geq 0$. By direct analysis we obtain that when $0<l<1$, the nonradial Weyl geodesics are convex curves (as seen from the origin) and they intersect some of the lines passing through the origin at two points (lying on the opposite sides of the origin). As a consequence some points in the plane are connected by two Weyl geodesics, enclosing the origin. Also the exponential mapping is onto $\mathbb{R}^{2} \backslash\{0\}$.

In dimension $n \geq 3$ we obtain by rotation continuous families of geodesics connecting two points on one line through the origin, with the origin between them. Hence we have "conjugate" points. It was shown in [W1 that similar to the riemannian case nonpositivity of Weyl sectional curvatures implies the absence of "conjugate" points.

In the case of nonpositive sectional curvatures, i.e., when $l>1$, the geodesics are concave as seen from the origin, and there is at most one geodesic connecting two points in $\mathbb{R}^{2} \backslash\{0\}$, and there may be none, i.e., the exponential map is one-to-one but it is not onto $\mathbb{R}^{2} \backslash\{0\}$.

Surprisingly the global dynamical behavior of the W-flow on the unit tangent bundle of $\mathbb{M}^{n}$ does not differ in the two cases. There are an attractor and a repellor, which are normally hyperbolic, and every orbit is asymptotic to them or is contained in one of them ([W1] $)$. 


\section{Proofs of Theorems 1.1 And 1.2}

Let us consider a Weyl connection on $\mathbb{R}^{n}\left(\mathbb{R}^{n} \backslash\{0\}\right)$ which factors onto the quotient manifold $\mathbb{T}^{n}\left(\mathbb{M}^{n}\right)$ and is integrable. It means that where the vector field $E$ does not vanish, the distribution of the subspaces orthogonal to $E$ is integrable. It is further equivalent to the condition that the bilinear form $\left\langle\nabla_{Y_{1}} E, Y_{2}\right\rangle$ is symmetric when restricted to the subspace orthogonal to $E$.

Theorem 4.1. If a Weyl connection on $\mathbb{R}^{n}\left(\mathbb{R}^{n} \backslash\{0\}\right)$ factors onto $\mathbb{T}^{n}\left(\mathbb{M}^{n}\right)$, $n \geq 3$, is integrable and has nonpositive sectional curvatures, then $E$ is constant in the toral case and in the case of $\mathbb{M}^{n} E=\frac{\alpha}{r} e_{r}$, with $|\alpha+1| \geq 1$.

Proof. Let us consider the vector field $F=|E|^{-2} E$. The bilinear form $\left\langle\nabla_{Y_{1}} F, Y_{2}\right\rangle$ is still symmetric on the subspace orthogonal to $E$, and in view of (2.5) it is actually symmetric on the whole space. Indeed it is sufficient to check that $\left\langle\nabla_{E} F, Y\right\rangle=$ $\left\langle\nabla_{Y} F, E\right\rangle$ for any vector $Y$ orthogonal to $E$, and we have

$$
\left\langle\nabla_{E} F, Y\right\rangle=\frac{1}{|E|^{2}}\left\langle\nabla_{E} E, Y\right\rangle, \quad\left\langle\nabla_{Y} F, E\right\rangle=-\frac{1}{|E|^{2}}\left\langle\nabla_{Y} E, E\right\rangle .
$$

It follows that the vector field $F$ is locally a gradient of a function $f$.

We proceed with a local analysis at a point $u_{0}$ where $F=\nabla f \neq 0$. In a neighborhood of $u_{0}$ the level sets of the function $f$ are smooth codimension 1 submanifolds. Observing that $X=|E|^{-1} E$ is the unit normal vector field of the level sets of $f$ and using Proposition 2.1, we get that the second fundamental form of a level set

$$
\left\langle\nabla_{Y} X, Y\right\rangle=|E|^{-1}\left\langle\nabla_{Y} E, Y\right\rangle=-|E|^{-1}\left\langle\nabla_{X} E, X\right\rangle=\nabla_{E} \frac{1}{|E|},
$$

where $Y$ is any unit vector tangent to the level set. In particular the level sets are umbilic and hence they are pieces of $(n-1)$-dimensional spheres or hyperplanes. Let us assume further that at our point $u_{0}$ the level set is a sphere of radius $r$. In a neighborhood of such a point the radius of the spherical level set $r=r(f)$ and its center $s=s(f)$ are locally smooth functions. Indeed (4.1) shows that $r$ is a local smooth function in $\mathbb{R}^{n}$ and we obtain $r(f)$ by composing $r$ with a smooth integral curve of $E$ parametrized by $f$. The smoothness of the center $s(f)$ follows. We can now parametrize a neighborhood of $u_{0}$ by $u=r(f) w+s(f)$, where $w$ belongs to the unit sphere $|w|^{2}=1$. Note that we can treat $w$ as a locally defined smooth vector field (the vector field of unit normals to the level sets of the function $f$ ). Let us consider an integral curve of $E, w=w(f)$. We get

$$
E=\frac{d u}{d f}=r^{\prime} w+r w^{\prime}+s^{\prime}=\left(r^{\prime}+\left\langle s^{\prime}, w\right\rangle\right) w+r w^{\prime}+s^{\prime}-\left\langle s^{\prime}, w\right\rangle w .
$$

Since $E$ is parallel to $w$ we conclude that

$$
E=\left(r^{\prime}+\left\langle s^{\prime}, w\right\rangle\right) w \quad \text { and } \quad r w^{\prime}+s^{\prime}-\left\langle s^{\prime}, w\right\rangle w=0 .
$$

Substituting the first equality into (4.1) we obtain

$$
\frac{1}{r}=-\frac{r^{\prime \prime}+\left\langle s^{\prime \prime}, w\right\rangle+\left\langle s^{\prime}, w^{\prime}\right\rangle}{\left(r^{\prime}+\left\langle s^{\prime}, w\right\rangle\right)^{2}},
$$

which yields using $r w^{\prime}=-s^{\prime}+\left\langle s^{\prime}, w\right\rangle w$

$$
r r^{\prime \prime}+\left(r^{\prime}\right)^{2}-\left(s^{\prime}\right)^{2}=-\left\langle r s^{\prime \prime}+2 r^{\prime} s^{\prime}, w\right\rangle-2\left\langle s^{\prime}, w\right\rangle^{2} .
$$


Since the left-hand side does not depend on $w$ (i.e., it is constant on level sets), the same must be true about the right-hand side. Setting $A=r s^{\prime \prime}+2 r^{\prime} s^{\prime}$ and $B=s^{\prime}$ we get that for any vector field $Y$ orthogonal to $E$

$$
0=\nabla_{Y}\left(\langle A, w\rangle+2\langle B, w\rangle^{2}\right)=\frac{1}{r}(\langle A, Y\rangle+4\langle B, w\rangle\langle B, Y\rangle) .
$$

We conclude that $A+4\langle B, w\rangle B$ is parallel to $w$. This implies that $A=B=0$. Hence $s^{\prime}=0$ and $r r^{\prime \prime}+\left(r^{\prime}\right)^{2}=0$. The integration of the last differential equation yields $f=c r^{2}+d$ for some constants $c, d$, and $F=\nabla f=2 c r e_{r}$ or equivalently $E=\frac{\alpha}{r} e_{r}$.

It remains to consider the case when the level sets are locally hyperplanes, where $E \neq 0$. It follows from (4.1) that $\left\langle\nabla_{Y} E, Y\right\rangle=0=\left\langle\nabla_{E} E, E\right\rangle$ for any vector $Y$ orthogonal to $E$. Further since by the integrability of the connection the quadratic form $\left\langle\nabla_{Y_{1}} E, Y_{2}\right\rangle$ is symmetric on the subspace orthogonal to $E$, we conclude that it must vanish there. By (2.5) we also have $\left\langle\nabla_{E} E, Y\right\rangle=-\left\langle\nabla_{Y} E, E\right\rangle$ for any vector $Y$ orthogonal to $E$. It follows that $\nabla E$ is skewsymmetric and hence $E$ must be a Killing vector field, i.e, $E(u)=H u+h$, where $H$ is a skewsymmetric matrix and $h$ is a constant vector. Clearly none of the Killing vector fields satisfies the equivariance condition (2.1), and only the constant field factors onto the torus.

It is obvious that Theorem 4.1 implies Theorem 1.1. In the case of $\mathbb{M}^{n}$ we can drop the assumption of integrability in Theorem 4.1, which is the content of Theorem 1.2.

Proof of Theorem 1.2. We introduce on $\mathbb{M}^{n}=\mathbb{S}^{1} \times \mathbb{S}^{n-1}=\left(\mathbb{R}^{n} \backslash\{0\}\right) / D$ the product riemannian metric $d^{2} s=\frac{d^{2} r}{r^{2}}+d^{2} \theta$, where $(r, \theta)$ are spherical coordinates in $\mathbb{R}^{n} \backslash\{0\}$. This metric has nonnegative sectional curvatures, and its Ricci curvature is positive except for the direction of $\mathbb{S}^{1}$, where it vanishes (this can be checked using (1.2) and (1.3)). Let $E$ be the tangent vector field on $\mathbb{M}^{n}$ defining the Weyl connection with respect to this metric. We now repeat the argument from the proof of Proposition 2.1.

For a plane $\Pi$ containing $E \neq 0$ we have by (1.3)

$$
\widehat{K}(\Pi)=K(\Pi)-\left\langle\nabla_{X} E, X\right\rangle-\left\langle\nabla_{Y} E, Y\right\rangle \leq 0,
$$

for $X=|E|^{-1} E$ and any unit vector $Y$ orthogonal to $E$. Averaging over all such vectors $Y$ we get from (4.4)

$$
\frac{1}{n-1}\left(\operatorname{Ric}(X, X)-\operatorname{div} E-(n-2)\left\langle\nabla_{X} E, X\right\rangle\right) \leq 0 .
$$

It follows that for the $C^{1}$ vector field $V=|E|^{n-2} E$ we have

$$
|E|^{n-2} \operatorname{Ric}(X, X)-\operatorname{div} V \leq 0 .
$$

We treat the left-hand side as a function defined on $\mathbb{M}^{n}$ (vanishing where $E=0$ ), and integrating it over $\mathbb{M}^{n}$ (with respect to the riemannian volume) we obtain $\int|E|^{n-2} \operatorname{Ric}(X, X) \leq 0$. Since the integrand is nonnegative it must vanish identically. As observed above, $\operatorname{Ric}(Z, Z)$ vanishes if and only if $Z$ is parallel to $\mathbb{S}^{1}$. We conclude that if the vector field $E$ does not vanish, then it must be parallel to $\mathbb{S}^{1}$.

To obtain the euclidean metric in $\mathbb{R}^{n} \backslash\{0\}$ we need to multiply our product metric by $r^{2}$. The vector field defining the Weyl connection with respect to the euclidean metric will be different from $E$, but it will still be a radial vector field. 
Now it is sufficient to recall Section 3, where we found all radial vector fields on $\mathbb{R}^{n} \backslash\{0\}$, which define Weyl connections on $\mathbb{M}^{n}$ with nonpositive sectional curvatures.

\section{REFERENCES}

[AW] R. Azencott, E.N. Wilson, Homogeneous manifolds with negative curvature, I, Trans. AMS 215 (1976), 323 - 362. MR0394507 (52:15308)

[F] G. B. Folland, Weyl manifolds, J. Diff. Geom. 4 (1970), 145 - 153. MR0264542 (41:9134)

[GR] G. Gallavotti, D. Ruelle, SRB states and nonequilibrium statistical mechanics close to equilibrium, Commun. Math. Phys. 190 (1997), 279 - 285. MR1489572 (99a:82057)

[G] P. Gauduchon, La 1-forme de torsion d'une variété hermitienne compacte, Math. Ann. 267 (1984), 495 - 518. MF 0742896 (87a:53101)

[R] D. Ruelle, Smooth dynamics and new theoretical ideas in nonequilibrium statistical mechanics, J. Stat. Phys. 95 (1999), 393 - 468. MR.1705592 (2000k:82054)

[W1] M.P. Wojtkowski, W-flows on Weyl manifolds and gaussian thermostats, J. Math. Pures. Appl 79 (2000), 953 - 974. MR1801870(2001k:37044)

[W2] M.P. Wojtkowski, Weyl manifolds and Gaussian thermostats, Proceedings of the International Congress of Mathematicians, Beijing 2002 III (2002), 511 - 523. MR1957557 (2004c:37155)

Department of Mathematics, University of Arizona, Tucson, Arizona 85721

E-mail address: maciejw@math.arizona.edu 